\title{
Effect of Endoscopic Gastroplication on the Genome-Wide Transcriptome in the Upper Gastrointestinal Tract
}

\author{
Nikkie van der Wielen ${ }^{1,2} \cdot$ Givan Paulus $^{3} \cdot$ Mark van Avesaat $^{1,4}$.

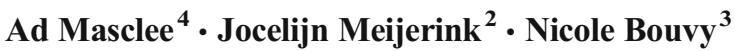

Published online: 13 September 2016

(C) The Author(s) 2016. This article is published with open access at Springerlink.com

\begin{abstract}
Background Bariatric surgery is an effective intervention strategy in obesity, resulting in sustained weight loss and a reduction of comorbidities. Gastroplication, using the articulating circular endoscopic stapler, was recently introduced as a transoral bariatric technique. This procedure reduces gastric volume and induced $34.9 \%$ of excess weight loss in the first year (Paulus et al. Gastrointest Endosc. 81(2):312-20, 3). The aim of the present study was to gain insight in the long-term effects and underlying mechanisms of gastroplication by investigating differences in the genome-wide gastric and duodenal transcriptome before and 1 year after intervention. Methods Ten morbidly obese patients (BMI $39.8 \pm 0.9 \mathrm{~kg} / \mathrm{m}^{2}$ $($ mean $\pm \mathrm{SEM})$ ) underwent gastroplication. Previous to the procedure and after 1 year, blood samples were taken, and mucosal biopsies were collected from the fundus, antrum and duodenum. Gene expression was measured using
\end{abstract}

Nikkie van der Wielen and Givan Paulus contributed equally to this work

Electronic supplementary material The online version of this article (doi:10.1007/s11695-016-2356-0) contains supplementary material, which is available to authorized users.

Jocelijn Meijerink

jocelijn.meijerink@wur.nl

Top Institute Food and Nutrition, Wageningen, The Netherlands

2 Division of Human Nutrition, Wageningen University, Wageningen, The Netherlands

3 Department of General Surgery, NUTRIM, Maastricht University Medical Center, Maastricht, The Netherlands

4 Division of Gastroenterology and Hepatology, Department of Internal Medicine, NUTRIM, Maastricht, University Medical Center, Maastricht, The Netherlands microarray analysis. Plasma adiponectin, $\mathrm{HbA} 1 \mathrm{c}$, IL- $1 \beta$, IL6 , IL-7, TNF- $\alpha$, IFN- $\gamma$, MCP-1, IL-8, TGF-1 and CRP levels were determined.

Results Downregulation of inflammatory genes and gene sets was observed in the fundus and duodenum 1 year after surgery. Gene expression of ghrelin and its activating enzyme GOAT were downregulated in the upper gastrointestinal tract. Patients showed a reduction in plasma $\mathrm{HbAlc}$ levels (from $6.17 \pm 0.51$ to $5.32 \pm 0.14 \%, p=0.004)$ and an increase of plasma adiponectin (from $16.87 \pm 3.67$ to $27.67 \pm 5.92 \mu \mathrm{g} / \mathrm{ml}$, $p=0.002$ ).

Conclusions Individuals undergoing gastroplication displayed a downregulation of inflammatory tone in the stomach and duodenum, which coincided with improved $\mathrm{HbAlc}$ and adiponectin levels. The reduction of inflammatory tone in the upper gastrointestinal tract may be a consequence of an improved metabolic health status or alternatively caused by the procedure itself.

Keywords Gastroplication · Transcriptome $\cdot$ Gene expression · Gastric tissue $\cdot$ Duodenum $\cdot \mathrm{HbA1c} \cdot$ Adiponectin $\cdot$ Immunity $\cdot$ Inflammation

\section{Introduction}

Bariatric surgery is the most effective medical option to achieve sustained weight loss in severe obesity. Besides traditional procedures such as laparoscopic Roux-en-Y gastric bypass (RYGB), vertical sleeve gastrectomy (VSG) and adjustable gastric banding (LAGB), less invasive options are available such as the (transorally placed) duodenal-jejunal bypass sleeve (DJBS). In general, these procedures lead to a loss of body fat, a reduction of comorbidities and improvement of long-term health risks. Remarkably, the mechanisms behind 
these outcomes are still poorly understood, and it is conceivable that these comprise different combinations of biological adaptations [1]. This is reflected in the markedly different immediate effects on glycaemic control following different procedures [2]. Recently, endoscopic gastroplication has become available as a new minimal invasive technique. The articulating circular endoscopic (ACE) stapler is used to reduce the volume of the stomach without removing tissue or bypassing other intestinal regions. For this procedure, no skin incisions are necessary; it is performed via a transoral route. This procedure results in a median $34.9 \%$ (IQR 17.8-46.6) loss of excess weight in the first year. Moreover, only mild adverse effects were reported so far [3]. Although several studies have described metabolic and anti-inflammatory effects of bariatric surgery at a molecular level, studies on these processes within the gastrointestinal (GI) tract are still limited. This holds particularly true for the upper GI tract, as most studies in this field have focussed on the mid or lower gastrointestinal tract [4-6]. Moreover, these studies concern effects of RYGB, a procedure extensively changing GI anatomy and physiology. The present study was undertaken to gain more insight in the long-term effects and underlying mechanisms of gastroplication in the upper GI tract: the stomach (fundus and antrum) and the duodenum, and to relate these to general health outcomes, including parameters of inflammation. To this end, transcriptome and gene set enrichment analysis was performed with biopsies obtained before and 1 year following gastroplication.

\section{Materials and Methods}

\section{ACE Stapler Study}

This study used biopsies and blood samples obtained from ten patients who were part of the first human ACE stapler study [3]. The Medical Ethical Committee of the Maastricht University Medical Center+ in the Netherlands (NCT02381340) approved the present study as a sub-study aiming to further unravel underlying mechanisms. Before inclusion, written informed consent was obtained from each participant. The inclusion criteria for the ACE stapler study are described in detail by Paulus et al. [3]. In brief, participants were 18 to 50 years old with a BMI of 40 to $45 \mathrm{~kg} / \mathrm{m}^{2}$ or 30 to $39.9 \mathrm{~kg} / \mathrm{m}^{2}$ in combination with one or more comorbidities expected to improve with weight loss. The ACE stapler was introduced into the stomach together with a thin endoscope. By applying vacuum to the gastric tissue, a large full-thickness (transmural) plication was drawn into the stapler head and fixed with a staple ring. Reduction of the stomach volume along the greater curvature was completed after creating a maximum of eight plications in the fundus and two additional plications in the antrum of the stomach. More details on the procedure were published previously [3]. Mucosal biopsies were taken from the fundus, antrum and duodenum with a standard forceps before starting the procedure. Afterwards, patients visited the outpatient clinic regularly and were stimulated to adhere to a healthy lifestyle. A follow-up endoscopy was planned 12 months after the procedure, at which the biopsy procedure was repeated. Biopsies were snap frozen in liquid nitrogen and stored at $-80{ }^{\circ} \mathrm{C}$ until analysis. Table 1 shows a brief overview of the characteristics of included patients.

\section{RNA Isolation and Microarray Processing}

RNA of the mucosal biopsies was isolated using TRIzol reagent (Life technologies, Bleiswijk, Netherlands) and further purified using the RNeasy micro kit (Qiagen, Venlo, Netherlands). RNA yield was measured with the Nanodrop ND-1000 Spectrophotometer, and the quality of the RNA samples was verified with an Agilent 2100 Bio analyser (Agilent Technologies, Amstelveen, Netherlands). One hundred nanogram of RNA was used for whole transcript cDNA synthesis (Affymetrix, Inc., Santa Clara, USA). Hybridization, washing and scanning of Affymetrix GeneChip Human Gene 1.1 ST arrays was carried out according to standard Affymetrix protocols.

\section{Microarray Analysis}

For the analysis of the microarray results, each location (i.e. fundus, antrum and duodenum) was analysed separately. Arrays were normalized using the robust multiarray average method [7, 8]. Probe sets were assigned to unique gene identifiers, in this case Entrez IDs. The probes on the arrays represent 19654 Entrez IDs [9]. Array data were analysed using MADMAX pipeline for statistical analysis of microarray data [10]. Quality control was performed, and all arrays met our criteria, except for the fundus and antrum arrays from participant 5, which were excluded. All data were filtered, and probe sets with

Table 1 Overview of characteristics of patients undergoing ACE stapler procedure. Measurements were performed at baseline and 1 year after the procedure. Ghrelin was measured after a 10 hour overnight fast

\begin{tabular}{llllll}
\hline & \multicolumn{2}{l}{ Baseline } & & \multicolumn{2}{l}{ One year } \\
\cline { 2 - 3 } & Mean & SEM & & Mean & SEM \\
\hline Age & 39 & 2 & & \\
Male/female ratio & $6: 4$ & & & \\
BMI $\left(\mathrm{kg} / \mathrm{m}^{2}\right)$ & 39.8 & 0.9 & & 33.4 & 0.9 \\
Excess weight loss $(\%)$ & & & 37.9 & 4.8 \\
Fasted active ghrelin level $(\mathrm{pg} / \mathrm{ml})$ & 46.5 & 5.9 & 63.4 & 5.2 \\
\hline
\end{tabular}


Table 2 The effect of ACE stapler treatment on fasted plasma levels of inflammatory and metabolic markers. Plasma levels were measured before the treatment (baseline) and 1 year after

\begin{tabular}{|c|c|c|c|c|c|c|c|}
\hline \multirow[t]{2}{*}{ Plasma marker } & \multirow[b]{2}{*}{ Unit } & \multirow{2}{*}{$\begin{array}{l}\text { Baseline } \\
\text { Mean }\end{array}$} & \multirow[b]{2}{*}{ SEM } & \multirow{2}{*}{$\begin{array}{l}\text { One year } \\
\text { Mean }\end{array}$} & \multirow[b]{2}{*}{ SEM } & \multicolumn{2}{|c|}{ Treatment effect } \\
\hline & & & & & & Difference & $p$ value \\
\hline Adiponectin & $\mu \mathrm{g} / \mathrm{ml}$ & 16.87 & 3.67 & 27.67 & 5.92 & 10.80 & $0.002 *$ \\
\hline $\mathrm{HbAlc}$ & $\%$ & 6.17 & 0.51 & 5.32 & 0.14 & -0.85 & $0.004 *$ \\
\hline IL-1 $\beta$ & $\mathrm{pg} / \mathrm{ml}$ & 1.42 & 0.06 & 1.37 & 0.08 & -0.05 & 0.244 \\
\hline IL-6 & $\mathrm{pg} / \mathrm{ml}$ & 10.90 & 1.83 & 7.41 & 1.80 & -3.49 & 0.069 \\
\hline IL-7 & $\mathrm{pg} / \mathrm{ml}$ & 11.52 & 1.24 & 10.43 & 2.01 & -1.09 & 0.180 \\
\hline TNF- $\alpha$ & $\mathrm{pg} / \mathrm{ml}$ & 2.14 & 0.10 & 2.00 & 0.14 & -0.14 & 0.118 \\
\hline IFN- $\gamma$ & $\mathrm{pg} / \mathrm{ml}$ & 2.54 & 0.27 & 2.23 & 0.25 & -0.32 & 0.099 \\
\hline MCP-1 & $\mathrm{pg} / \mathrm{ml}$ & 60.70 & 7.33 & 46.68 & 7.74 & -14.02 & 0.088 \\
\hline IL-8 & $\mathrm{pg} / \mathrm{ml}$ & 7.03 & 1.03 & 6.55 & 1.44 & -0.48 & 0.455 \\
\hline LAP/TGF-1 & $\mathrm{ng} / \mathrm{ml}$ & 3.11 & 0.35 & 3.24 & 0.36 & 0.13 & 0.393 \\
\hline CRP & $\mathrm{mg} / 1$ & 12.62 & 5.66 & 8.78 & 2.75 & -3.85 & 0.248 \\
\hline
\end{tabular}

expression values above 20 in at least 5 arrays were included for further analysis. These data were used for gene set enrichment analysis (GSEA; www.broadinstitute. org/gsea [11]) in MADMAX. Gene sets with a false discovery rate $(F D R)<0.25$ were considered significantly enriched. The gene set enrichment analysis was visualized using the enrichment plugin in Cytoscape with conservative filtering $(p<0.001$ and FDR $q<0.05)$. For further analysis of individual genes, a cut-off of IQR $>0.25$ was used to filter out genes that showed no variation between the samples; Intensity-Based Moderated T-statistics (IBMT) was used to assess significant differences with $p$ value $<0.05$.

\section{Plasma Measurements}

Blood samples were collected in EDTA-coated tubes, centrifuged and stored at $-80 \mathrm{C}$ until analysis. The measurement of plasma adiponectin and cytokine levels was performed using an in-house developed and validated
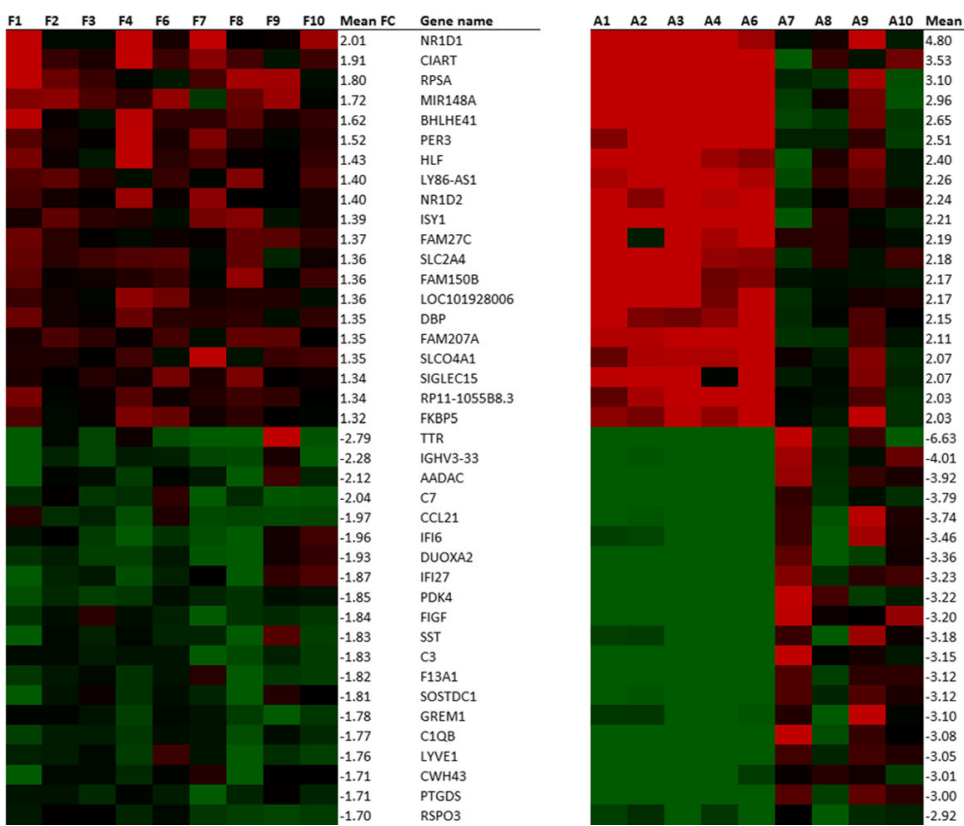

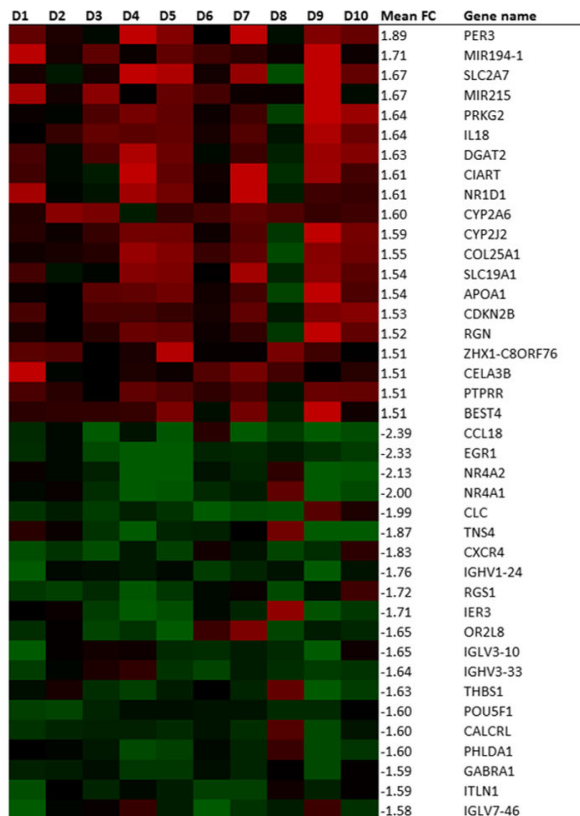

from the mean fold change (FC) of the top regulated genes by the treatment, signal log ratios are displayed to show inter-individual differences

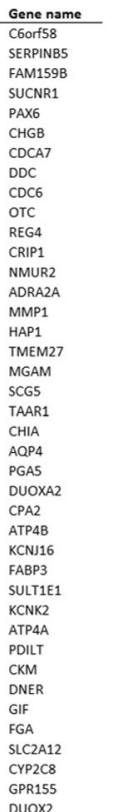

Fig. 1 Expression of most highly significantly upregulated and downregulated genes in fundus, antrum and duodenum, respectively. Green is a signal $\log$ ratio of -2 , and red is a signal log ratio of 2. Apart 
multiplex immunoassay (Laboratory of Translational Immunology, University Medical Center Utrecht, the Netherlands) based on Luminex technology (xMAP, Luminex, Austin, USA). The assay was performed as described previously [12]. Using heteroblock (Omega Biologicals, Bozeman, USA), aspecific heterophilic immunoglobulins were preabsorbed. Acquisition was performed with the Biorad FlexMAP3D (Biorad laboratories, Hercules, USA) in combination with XPONENT software version 4.2 (Luminex, Austin, USA). Data was analysed by 5-parametric curve fitting using Bio-Plex Manager software, version 6.1.1 (Biorad laboratories, Hercules,

\section{a}

\section{Fundus}

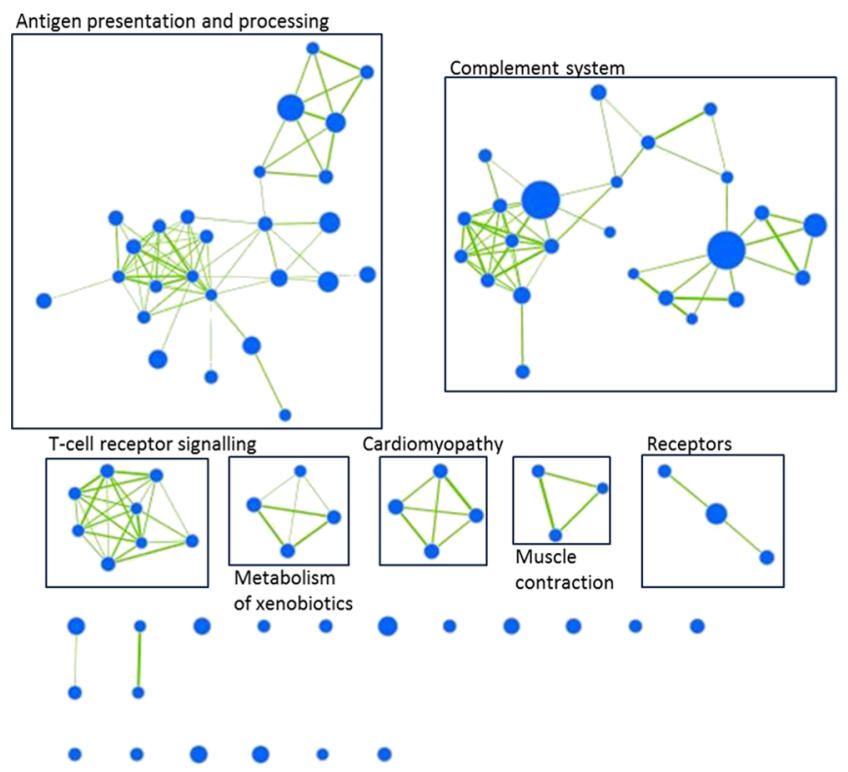

C

Duodenum

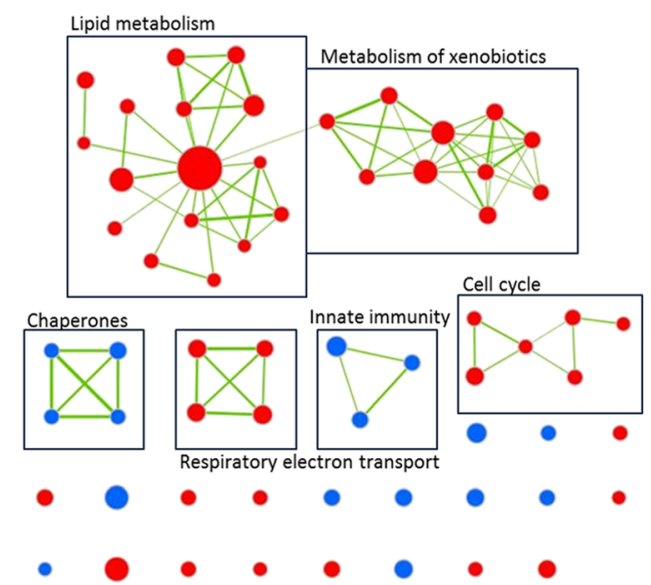

Fig. 2 Gene set enrichment analysis of the fundus, antrum and duodenum. Each node indicates a conservative filtered gene set $(p<0.001$ and FDR $\mathrm{q}<0.05)$, and the connecting lines indicate
USA). HbA1c levels were determined routinely at the Department of Clinical Chemistry of the Maastricht University Medical Center. Active ghrelin was measured using an established in-house radioimmunoassay (Millipore, Massachusetts, USA).

\section{Statistical Analysis}

Statistical analyses were performed using Prism 5.0 (GraphPad Software, Inc. La Jolla, USA). The effects of treatment on plasma levels of inflammatory markers and adiponectin were tested by paired $t$ tests for normally

b

Antrum

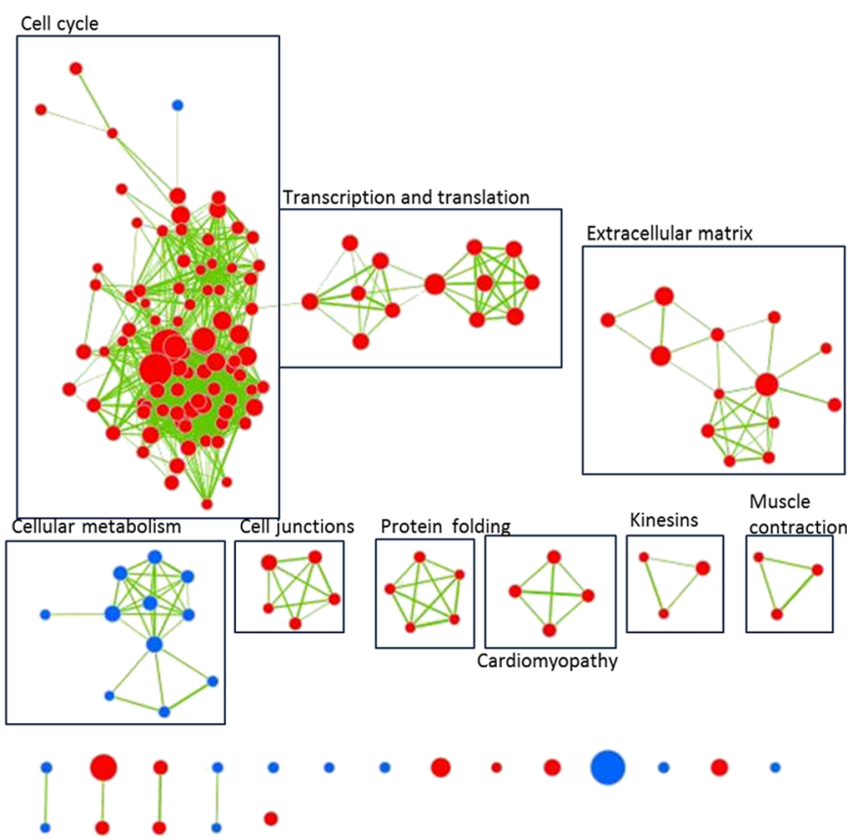

overlapping genes between the nodes/gene sets. Red is enriched; blue is depleted gene sets 
distributed variables and Wilcoxon's signed rank tests for non-normally distributed variables. Spearman's rank correlation coefficient analysis was performed to investigate the association between changes in biochemical parameters with changes in mRNA expression (signal log ratio). A $p$ value $<0.05$ was considered statistically significant. Data are presented as mean with standard error of the mean (SEM). Statistical analysis of transcriptome data was described above.

\section{Results}

\section{Effects of Gastroplication on Systemic Metabolic and Inflammatory Parameters One Year After Intervention}

Plasma levels of glycated haemoglobin, adiponectin and several pro-inflammatory mediators before and after intervention are shown in Table 2. Significant changes were found for adiponectin and HbA1c. Adiponectin showed a 1.64-fold increase $(p=0.002)$ in the patients who underwent ACE stapler treatment. Glycated haemoglobin (HbA1c) was significantly decreased $(p=0.004)$ by the treatment. Plasma IL-6 showed a tendency to decrease following ACE stapler treatment by a factor 1.47. MCP1 levels also showed a decrease (1.3-fold), but this effect did not reach statistical significance.

\section{Effects on Tissue Gene Expression Mainly Relate to Inflammatory Pathways}

Gene expression changes 1 year after intervention compared to baseline were analysed for different locations of the upper gastrointestinal tract, namely fundus, antrum and duodenum. After intervention, 727 genes (259 upregulated, 468 downregulated) were significantly changed in the fundus, 1846 (951 upregulated, 895 downregulated) in the antrum and 921 genes (480 upregulated, 441 downregulated) in the duodenum. The top 20 upregulated and downregulated genes in all three locations are shown in Fig. 1. In both fundus and duodenum, a considerable number of downregulated genes have been associated with immunity and inflammatory pathways. In the fundus, the expression of immune-related genes like IGHV3-33, C7, CCL21, IFI6, IFI27, C1QB was downregulated, and CCL18, CLC, CXCR4, IGHV1-24, RSG1, IGLV3-10, IGHV3-33, IGLV7-46 were downregulated in the duodenum. In the antrum, there was an upregulation of some neuroendocrine-associated genes, namely $P A X 6, C H G B$, SCG5.

\section{Gene Set Enrichment Analysis Reveals Potential Processes Involved}

To gain more insight into the processes changed 1 year following the stomach volume reduction procedure, gene
Fig. 3 Changes in gene expression of ghrelin and MBOAT4 and their correlation to plasma ghrelin. a The graphs show RMA normalized intensities of microarray data at baseline and 1 year after gastroplication in biopsies of fundus, antrum and duodenum. Fold changes are indicated on top of the bars, significant changes are marked with asterisk $(p<0.05)$. All graphs show mean and SEM. b Correlation between signal $\log$ ratios of ghrelin (GHRL) and MBOAT4 in the fundus with changes in plasma active ghrelin levels measured in a fasted state before and 1 year after the procedure a

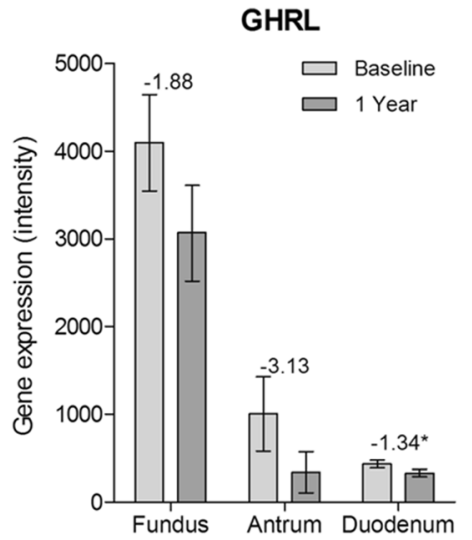

b

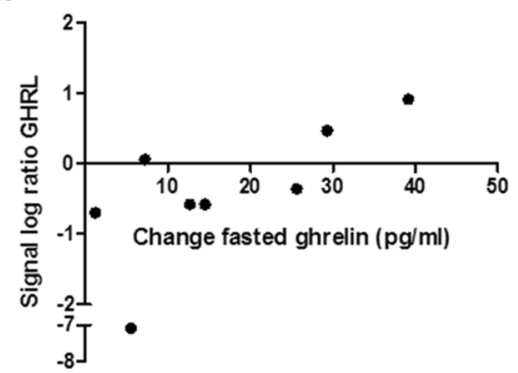

MBOAT4
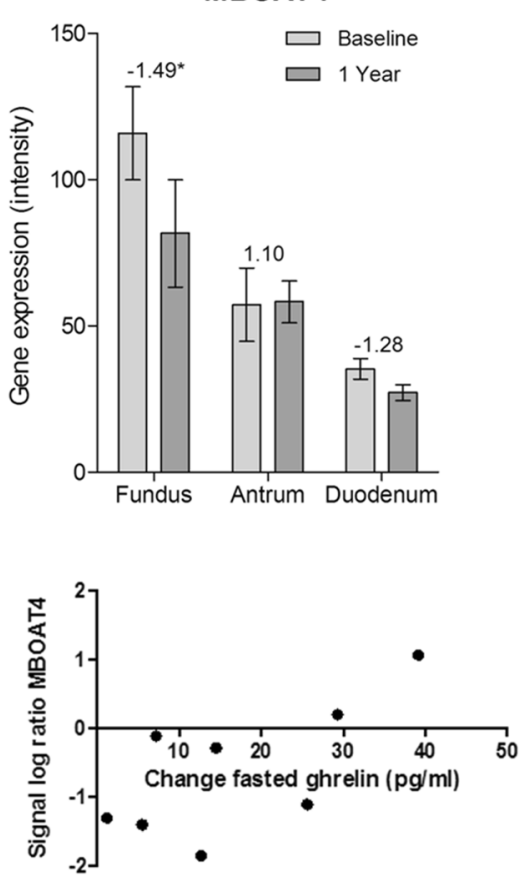
set enrichment analysis (GSEA) was performed. This computational method uses molecular signatures to associate changes in gene expression with known biological processes. Analysis resulted in 236 (2 upregulated, 234 downregulated) enriched gene sets for fundus, 546 (474 upregulated, 72 downregulated) enriched gene sets for antrum and 253 (182 upregulated, 71 downregulated) enriched gene sets for duodenum. In the antrum, more gene sets were upregulated, whereas in the fundus, most gene sets were downregulated (Fig. 2). Of these downregulated gene sets in the fundus, many were related to immune responses, mostly to the complement system, presentation and recognition of antigens (self or pathogenic) and $\mathrm{T}$ cell receptor signalling. Also in the duodenum, some of the downregulated gene sets were related to the innate immunity. In the antrum, cell cycle related gene sets were strongly enriched. In the duodenum, the enrichment analysis showed also a slight upregulation of cell cycle processes. Here, more metabolic pathways were apparently upregulated, including those associated with 'fat digestion and absorption' and 'metabolism of lipids and lipoproteins'. All gene sets are specified in Table S1.

\section{Changes in Gastrointestinal Hormone Expression}

Being one of the main gastric hormones, ghrelin is not only involved in appetite regulation but also in immunity [13]. Two genes related to ghrelin were significantly changed in specific locations of the GI tract (Fig. 3a). In the fundus, there was a downregulation of MBOAT4 $(\mathrm{FC}=-1.49)$, the gene encoding the ghrelin-activating enzyme GOAT4. Furthermore, there was a trend for downregulation of ghrelin $(G H R L)$ expression itself in the fundus $(\mathrm{FC}=-1.88, p=0.19)$ and antrum $(\mathrm{FC}=-3.13, p=0.11)$ and a significant downregulation in the duodenum $(\mathrm{FC}=-1.34)$.

In the fundus, the ghrelin gene expression (GHRL) was positively correlated to fasted plasma changes of active ghrelin (Spearman correlation coefficient $=0.826, p=0.015$ ), and for MBOAT4 expression, there was a tendency for correlation (Spearman correlation coefficient $=0.69, p=0.069)($ Fig. 3b). Although the correlation between changes in ghrelin and GHRL expression seems to be influenced by a single patient, excluding this patient still resulted in a significant correlation.

\section{Conclusions}

Results of the present study add important physiological background information to the clinical outcomes observed in patients after undergoing ACE stapler gastroplication. Our plasma analyses revealed beneficial effects on $\mathrm{HbA} 1 \mathrm{c}$ and adiponectin levels 1 year after surgery, indicating an improvement of glycaemic control and a favourable shift in adipose tissue mass and/or inflammatory status, respectively. This rise in plasma adiponectin and reduction of $\mathrm{HbAlc}$ is in line with previous studies in which a loss of excess body fat was achieved, including those involving other surgical and non-surgical weight loss interventions [14-17]. We also found tendencies for reduced plasma IL-6 and MCP-1 levels, 1 year after intervention. Reduced levels of inflammatory markers are generally assumed to result from a reduction of visceral fat mass in particular, which plays a major role in the low grade inflammatory state associated with obesity [18]. The total number of patients in our study population is quite small, and it is conceivable that statistical significance might have been reached with a larger patient group. Several other studies have found significant reductions in inflammatory markers like CRP, MCP-1 and IL-6 after bariatric surgery, while others did not find such an effect [19-21].

To our knowledge, this study is the first to analyse longterm whole transcriptome changes in the upper gastrointestinal tract after a new transoral bariatric procedure. Gastroplication reduces gastric volume without altering intestinal anatomy, as is the case with RYGB. In contrast to bypass surgery, exposure of the intestinal epithelium to nutrients and their metabolites is largely maintained after this endoscopic gastroplication. At the same time, small changes related to different GI transit characteristics or changes in the microbiome might still occur.

Analysis of the large amount of data using unbiased transcriptome analysis clearly pointed towards a reduction in inflammatory tone in the fundus and duodenum tissues as manifested by the downregulation of a wide variety of inflammation-related gene sets. The downregulated gene sets in the fundus were mostly related to innate immunity, and particularly associated with downregulation of the complement system, presentation and recognition of antigens (self or pathogenic), IFN- $\gamma$ signalling and $\mathrm{T}$ cell receptor signalling. In the duodenum, the main downregulated gene set was associated with the complement system. Moreover, the top 20 highly changed genes in these locations also suggest notable downregulation of many immune-related processes, of which several were related to chemokines, complement system, interferon signalling and immunoglobulins. These results coincided with the weight loss, improvement of $\mathrm{HbAlc}$ levels and decrease of whole-body inflammatory tone in these patients.

Based on the present study, we cannot establish whether the apparent reduction of inflammatory tone in the upper GI tract has a predominantly local cause, i.e. due to a changed foodintake pattern or digestion process, or whether it is related to a reduction of low-grade systemic inflammation due to the reduction of body fat mass.

Increasing evidence points to a link between intestinal inflammation status in general, obesity and (or) diabetes. In obesity, increased innate cell densities, among which 
macrophages, natural killer cells and $\mathrm{T}$ cells, especially the proportion of cytotoxic CD8 T cells, have been observed in the jejunal epithelium. These epithelial $\mathrm{T}$ cells were found to be associated with local and systemic comorbidities. Furthermore, isolated $\mathrm{T}$ cells from obese patients decreased insulin sensitivity of epithelial cells in vitro [22]. Another study reported that diet-induced weight loss resulted in a downregulation of inflammatory pathways and inflammatory cytokines IL-8, TNF- $\alpha$, MCP-1 and IL-1 $\beta$, in recto-sigmoid mucosal tissue [23]. Moreover, increased intestinal inflammatory gene expression of TNF- $\alpha$, IL-6, ICAM and PTGS- 2 was found in insulin-resistant obese patients compared to noninsulin-resistant obese patients, suggesting that intestinal inflammation is involved in diabetes during obesity [24, 25]. A prominent feature of the immune system in the gastrointestinal tract is to provide adequate protection without stimulating excessive inflammation, thereby maintaining a fine balance $[26,27]$. A pro-inflammatory immune status of the gastrointestinal tract in obese patients might be protective against increased luminal challenges associated within obesity but deteriorating for insulin resistance [22]. Furthermore, this proinflammatory status might be linked to the increased prevalence of inflammatory bowel disease and cancer in obese patients [28-30]. In summary, we can only speculate whether the reduced inflammatory microenvironment in gastric and duodenal tissue found after gastroplication can be considered as a positive or negative outcome.

In the antral tissue, gene sets related to cell cycle processes and extracellular matrix were increased. This might be explained by dilation of the stomach, a common observation after gastric volume reduction [31-34]. While gastric volume was not quantified, we perceived the stomach as larger at 1-year follow-up than immediately after gastroplication.

An interesting observation was that the mean gene expression of ghrelin and the enzyme GOAT, responsible for ghrelin acylation, decreased after gastroplication in some of the tissues. At the same time, plasma fasted active ghrelin was increased, and there was a positive correlation between the gene expression of ghrelin and plasma values of active ghrelin. Ghrelin is one of the most prominent hormones secreted from the upper gastrointestinal tract and does not only play a role in appetite regulation but also in inflammation [13]. Consistent with our results, in RYGB patients, significant lower levels of jejunal ghrelin gene expression have been reported after 10 months [6]. Furthermore, GOAT mRNA expression and GOATpositive cell numbers were lower in a non-obese group compared to morbidly obese patients, although no changes in jejunal ghrelin expression were detected [35]. Moreover, more ghrelin positive cells were found in the stomach of morbidly obese and overweight patients compared to healthy normal weight controls [36, 37], which might indicate that with weight loss, the number of ghrelin-releasing cells will decrease. The discrepancy with ghrelin expression in the gastrointestinal tract and plasma ghrelin values might be explained by a reduced secretory activity of (a higher number of) ghrelin producing cells in obesity, as suggested by Widmayer et al. [36]. However, within our patients, there was a positive correlation between its gene expression and plasma levels, indicating that upregulated expression of ghrelin in the fundus was associated with greater increase in fasted ghrelin levels and downregulated expression with a smaller increase 1 year after gastroplication. The underlying cause of the observed changes cannot be pinpointed in our study. It is possible that the changes in ghrelin are dependent on the surgical procedure, which takes place at the main site of ghrelin secretion. Furthermore, the implications of these changes in ghrelin are not fully understood and need further investigation to crystallize the underlying mechanism and to explore the potential of these changes in obesity treatment.

There are some strengths and limitations to this study. The within-person measurement of changes in gastrointestinal gene expression is unique as most studies in this field are observational. By applying a prospective design, we were able to perform paired analysis and look specifically for changes induced by the gastroplication treatment instead of comparing obese subjects with lean controls. Whole transcriptome analysis enabled us to investigate changes in an unbiased manner. One of the limitations of this study is that it was not powered to find differences in inflammatory markers. Therefore, the inclusion of more patients could have strengthened the study. Furthermore, a control group on a lifestyle intervention program could help differentiate between weight loss effects and strictly procedural effects.

This study presents the long-term effects of a new transoral gastroplication treatment in morbidly obese patients. We show that this recently developed ACE stapler procedure was not only effective in reducing body weight as presented before [3], but also improved glycated haemoglobin levels and increased plasma adiponectin. Furthermore, whole transcriptome analysis suggested a marked downregulation of inflammatory gene sets in both the fundus and duodenum, coinciding with changes in plasma cytokines. Moreover, gene expression of ghrelin and its activating enzyme GOAT were reduced after gastroplication. The apparent reduction of inflammatory tone in the upper GI tract may be a consequence of an improved metabolic health status as associated with weight loss, or alternatively caused by the procedure itself.

In conclusion, this new transoral gastroplication treatment which induced significant weight loss and 
improved plasma levels of adiponectin and glycated haemoglobin coincides with a reduced inflammatory tone in the upper GI tract. The clinical relevance of our findings remains to be established, as there is still limited knowledge on the role of inflammatory pathways in the upper GI tract in obesity.

Acknowledgments Multiplex immunoassays were in-house developed, validated and performed by the Multiplex core facility of the Laboratory for Translational Immunology (LTI) of the University Medical Center Utrecht.

\section{Compliance with Ethical Standards}

Financial Disclosure Part of this study was jointly funded by Top Institute Food and Nutrition and BaroSense, Inc.

Conflict of Interest Two authors have received funding from TI Food and Nutrition, a public-private partnership on pre-competitive research in food and nutrition. The other authors have declared that no competing interests exist.

Ethical Statement and Consent Statement Informed consent was obtained from all individual participants included in the study.

All procedures performed were in accordance with the ethical standards of the institutional and/or national research committee and with the 1964 Helsinki declaration and its later amendments or comparable ethical standards.

Open Access This article is distributed under the terms of the Creative Commons Attribution 4.0 International License (http:// creativecommons.org/licenses/by/4.0/), which permits unrestricted use, distribution, and reproduction in any medium, provided you give appropriate credit to the original author(s) and the source, provide a link to the Creative Commons license, and indicate if changes were made.

\section{References}

1. Sweeney TE, Morton JM. Metabolic surgery: action via hormonal milieu changes, changes in bile acids or gut microbiota? A summary of the literature. Best Pract Res Clin Gastroenterol. 2014;28(4): $727-40$.

2. Li JF, Lai DD, Ni B, Sun KX. Comparison of laparoscopic Rouxen-Y gastric bypass with laparoscopic sleeve gastrectomy for morbid obesity or type 2 diabetes mellitus: a meta-analysis of randomized controlled trials. Can J Surg. 2013;56(6):E158-64.

3. Paulus GF, Verlaan T, Mathus-Vliegen EMH, Veldhuyzen EAML, Conchillo JM, Fockens P, et al. Endoscopic gastric volume reduction with a novel articulating plication device is safe and effective in the treatment of obesity. Gastrointest Endosc. 2015;81(2):312-20.

4. Furet JP, Kong LC, Tap J, Poitou C, Basdevant A, Bouillot JL, et al. Differential adaptation of human gut microbiota to bariatric surgery-induced weight loss: links with metabolic and low-grade inflammation markers. Diabetes. 2010;59(12):3049-57.

5. Casselbrant A, Elias E, Fandriks L, Wallenius V. Expression of tight-junction proteins in human proximal small intestinal mucosa before and after Roux-en-Y gastric bypass surgery. Surg Obes Relat Dis. 2015;11(1):45-53.

6. Rhee NA, Wahlgren CD, Pedersen J, Mortensen B, Langholz E, Wandall EP, et al. Effect of Roux-en-Y gastric bypass on the distribution and hormone expression of small-intestinal enteroendocrine cells in obese patients with type 2 diabetes. Diabetologia. 2015.

7. Bolstad BM, Irizarry RA, Astrand M, Speed TP. A comparison of normalization methods for high density oligonucleotide array data based on variance and bias. Bioinformatics. 2003;19(2):185-93.

8. Irizarry RA, Bolstad BM, Collin F, Cope LM, Hobbs B, Speed TP. Summaries of Affymetrix GeneChip probe level data. Nucleic Acids Res. 2003;31(4):e15.

9. Dai MH, Wang PL, Boyd AD, Kostov G, Athey B, Jones EG, et al. Evolving gene/transcript definitions significantly alter the interpretation of GeneChip data. Nucleic Acids Res. 2005;33(20).

10. Lin K, Kools H, de Groot PJ, Gavai AK, Basnet RK, Cheng F, et al. MADMAX - management and analysis database for multiple $\sim$ omics experiments. J Integr Bioinform. 2011;8(2):160.

11. Subramanian A, Tamayo P, Mootha VK, Mukherjee S, Ebert BL, Gillette MA, et al. Gene set enrichment analysis: a knowledgebased approach for interpreting genome-wide expression profiles. Proc Natl Acad Sci U S A. 2005;102(43):15545-50.

12. de Jager W, Prakken BJ, Bijlsma JW, Kuis W, Rijkers GT. Improved multiplex immunoassay performance in human plasma and synovial fluid following removal of interfering heterophilic antibodies. J Immunol Methods. 2005;300(1-2):124-35.

13. Prodam F, Filigheddu N. Ghrelin gene products in acute and chronic inflammation. Arch Immunol Ther Exp. 2014;62(5):369-84.

14. Yang WS, Lee WJ, Funahashi T, Tanaka S, Matsuzawa Y, Chao $\mathrm{CL}$, et al. Weight reduction increases plasma levels of an adiposederived anti-inflammatory protein, adiponectin. J Clin Endocrinol Metab. 2001;86(8):3815-9.

15. Lang HF, Chou CY, Sheu WH, Lin JY. Weight loss increased serum adiponectin but decreased lipid levels in obese subjects whose body mass index was lower than $30 \mathrm{~kg} / \mathrm{m}(2)$. Nutr Res. 2011;31(5):37886.

16. Penn L, White M, Lindstrom J, den Boer AT, Blaak E, Eriksson JG, et al. Importance of weight loss maintenance and risk prediction in the prevention of type 2 diabetes: analysis of European diabetes prevention study RCT. PLoS One. 2013;8(2):e57143.

17. Baskota A, Li S, Dhakal N, Liu G, Tian H. Bariatric surgery for type 2 diabetes mellitus in patients with BMI $<30 \mathrm{~kg} / \mathrm{m} 2$ : a systematic review and meta-analysis. PLoS One. 2015;10(7):e0132335.

18. Ghigliotti G, Barisione C, Garibaldi S, Fabbi P, Brunelli C, Spallarossa $\mathrm{P}$, et al. Adipose tissue immune response: novel triggers and consequences for chronic inflammatory conditions. Inflammation. 2014;37(4):1337-53.

19. Arismendi E, Rivas E, Agusti A, Rios J, Barreiro E, Vidal J, et al. The systemic inflammome of severe obesity before and after bariatric surgery. PLoS One. 2014;9(9):e107859.

20. Mallipedhi A, Prior SL, Barry JD, Caplin S, Baxter JN, Stephens JW. Changes in inflammatory markers after sleeve gastrectomy in patients with impaired glucose homeostasis and type 2 diabetes. Surg Obes Relat Dis. 2014;10(6):1123-8.

21. de Jonge C, Rensen SS, D’Agnolo HM, Bouvy ND, Buurman WA, Greve JW. Six months of treatment with the endoscopic duodenaljejunal bypass liner does not lead to decreased systemic inflammation in obese patients with type 2 diabetes. Obes Surg. 2014;24(2): $337-41$.

22. Monteiro-Sepulveda M, Touch S, Mendes-Sa C, Andre S, Poitou C, Allatif $\mathrm{O}$, et al. Jejunal $\mathrm{T}$ cell inflammation in human obesity correlates with decreased enterocyte insulin signaling. Cell Metab. 2015;22(1):113-24.

23. Pendyala S, Neff LM, Suarez-Farinas M, Holt PR. Diet-induced weight loss reduces colorectal inflammation: implications for colorectal carcinogenesis. Am J Clin Nutr. 2011;93(2):234-42.

24. Veilleux A, Mayeur S, Berube JC, Beaulieu JF, Tremblay E, Hould FS, et al. Altered intestinal functions and increased local 
inflammation in insulin-resistant obese subjects: a gene-expression profile analysis. BMC Gastroenterol. 2015;15:119.

25. Veilleux A, Grenier E, Marceau P, Carpentier AC, Richard D, Levy E. Intestinal lipid handling: evidence and implication of insulin signaling abnormalities in human obese subjects. Arterioscler Thromb Vasc Biol. 2014;34(3):644-53.

26. Hunt RH, Camilleri M, Crowe SE, El-Omar EM, Fox JG, Kuipers EJ, et al. The stomach in health and disease. Gut. 2015;64(10): 1650-68.

27. Cheroutre H, Lambolez F, Mucida D. The light and dark sides of intestinal intraepithelial lymphocytes. Nat Rev Immunol. 2011;11(7):445-56.

28. Versini M, Jeandel PY, Rosenthal E, Shoenfeld Y. Obesity in autoimmune diseases: not a passive bystander. Autoimmun Rev. 2014;13(9):981-1000.

29. Bardou M, Barkun AN, Martel M. Obesity and colorectal cancer. Gut. 2013;62(6):933-47.

30. Gonzalez CA, Sala N, Rokkas T. Gastric cancer: epidemiologic aspects. Helicobacter. 2013;18(Suppl 1):34-8.

31. Safatle-Ribeiro AV, Petersen PA, Pereira Filho DS, Corbett $\mathrm{CE}$, Faintuch J, Ishida R, et al. Epithelial cell turnover is increased in the excluded stomach mucosa after Roux-en-Y gastric bypass for morbid obesity. Obes Surg. 2013;23(10): 1616-23.

32. Iannelli A, Schneck AS, Hebuterne X, Gugenheim J. Gastric pouch resizing for Roux-en-Y gastric bypass failure in patients with a dilated pouch. Surg Obes Relat Dis. 2013;9(2): 260-7.

33. van Wezenbeek MR, Smulders JF, de Zoete JP, Luyer MD, van Montfort G, Nienhuijs SW. Long-Term Results of Primary Vertical Banded Gastroplasty. Obes Surg. 2014.

34. Baumann T, Grueneberger J, Pache G, Kuesters S, Marjanovic G, Kulemann B, et al. Three-dimensional stomach analysis with computed tomography after laparoscopic sleeve gastrectomy: sleeve dilation and thoracic migration. Surg Endosc. 2011;25(7):2323-9.

35. Ritze Y, Hengelhaupt C, Bardos G, Ernst B, Thurnheer M, D'Haese $\mathrm{JG}$, et al. Altered intestinal neuroendocrine gene expression in humans with obesity. Obesity. 2015.

36. Widmayer P, Kuper M, Kramer M, Konigsrainer A, Breer H. Altered expression of gustatory-signaling elements in gastric tissue of morbidly obese patients. Int J Obes. 2012;36(10):1353-9.

37. Maksud FA, Alves JS, Diniz MT, Barbosa AJ. Density of ghrelinproducing cells is higher in the gastric mucosa of morbidly obese patients. Eur J Endocrinol. 2011;165(1):57-62. 